Revue italienne d'études françaises

Littérature, langue, culture

6 | 2016

Les romanciers oubliés des années Trente

\title{
"Méfiez-vous de celui qui veut mettre de l'ordre" : (in)achèvement et imperfection chez Isabelle de Charrière
}

Paola Perazzolo

\author{
(2) OpenEdition \\ Journals \\ Édition électronique \\ URL : http://journals.openedition.org/rief/1224 \\ DOI : 10.4000/rief.1224 \\ ISSN : 2240-7456 \\ Éditeur \\ Seminario di filologia francese
}

Référence électronique

Paola Perazzolo, " "Méfiez-vous de celui qui veut mettre de l'ordre" : (in)achèvement et imperfection chez Isabelle de Charrière », Revue italienne d'études françaises [En ligne], 6 | 2016, mis en ligne le 15 décembre 2016, consulté le 10 décembre 2020. URL : http://journals.openedition.org/rief/1224 ; DOI : https://doi.org/10.4000/rief.1224

Ce document a été généré automatiquement le 10 décembre 2020.

\section{(c) (1) (9)}

Les contenus de la RIEF sont mis à disposition selon les termes de la Licence Creative Commons Attribution - Pas d'Utilisation Commerciale - Pas de Modification 4.0 International. 


\title{
"Méfiez-vous de celui qui veut mettre de l'ordre" : (in)achèvement et imperfection chez Isabelle de Charrière
}

\author{
Paola Perazzolo
}

Méfiez-vous de celui qui veut mettre de l'ordre. Ordonner, c'est toujours se rendre le maître des autres en les gênant. ${ }^{1}$

L'inachèvement n'est pas rupture. Il n'est pas non plus provocation. Il est simple refus de cette clôture par laquelle tout s'achève, vient à chef, selon l'étymologie du mot, se soumet à l'autorité du raisonnable, au nom d'une philosophie triomphante. ${ }^{2}$

1 Qualifiée par A. Rivara de "pratique sans théorie "³, l'incomplétude sous-tend de façon importante la production romanesque du XVIII ${ }^{\mathrm{e}}$ siècle: en adhésion aux préceptes aristotéliciens de la création de fables nécessitant un début, un développement et une fin, les réflexions théoriques ne cessent d'attester un souci d'achèvement souvent démenti dans la pratique. Des conditions d'écriture influencées par la censure et des modalités d'édition basées sur une notion de «droits des auteurs " qui reste pendant longtemps assez floue ${ }^{4}$ motivent la récurrence de publications par tranches, de romans in-finis relevant de l'esthétique de la liste ou de la série ainsi que l'existence de nombreuses "suites" et «continuations ». Tout au long du siècle, la reprise d'un texte reste aussi courante que son «imitation, réécriture, translation, toutes pratiques valorisées par la tradition $»^{5}$.

2 C'est dans cette tradition que s'insère également Isabelle de Charrière. "Salonnière virtuelle $»^{6}$ par son réseau européen et femme des Lumières sceptique et libre d'esprit, elle aussi fait preuve, dans sa production romanesque, d'un refus systématique de la 
clôture. La plupart de ses ouvrages manquent en effet de dénouement. Ce manque relève toutefois plus de l'inachèvement que de l'incomplétude, d'après la distinction rappelée par Gilles Louÿs, d'après lequel «si le concept d'inachèvement inclut nécessairement l'idée d'incomplétude, la réciproque n'est pas vraie, puisque nombre de romans de cette période doivent leur incomplétude à des pratiques qui ne sont ni accidentelles, ni exceptionnelles, mais qui illustrent au contraire l'état normal du genre $»^{7}$. Chez la Dame du Pontet, cette difficulté de "venir à chef » serait plus imputable à un choix poétique ou à une forme d'autocensure qu'à l'adhésion aux pratiques éditoriales évoquées, comme elle l'explicite dans sa correspondance en répondant à D'Oleyres qui insistait pour lire la suite des Lettres Neuchâteloises: «D'ailleurs, j'aurois peut-être encore moins de talent pour les dénouements que pour le reste. Les tristes sont tristes, et les heureux sont fort sujets à être plats $»^{8}$.

3 Exception faite pour Caliste (1787), suite des Lettres écrites de Lausanne relatant l'amour malheureux de William et de l'héroïne éponyme, les premiers romans - les Lettres Neuchâteloises (1784), les Lettres de Mistriss Henley (1784) et la première partie des Lettres écrites de Lausanne (1785) - affichent ce qu'on pourrait définir comme un (in)achèvement qui est structurel dans la mesure où le récit est complet et achevé dans sa forme tout en demeurant «ouvert». Différemment, l'absence de la fin se fait souvent matérielle dans les ouvrages de la période révolutionnaire, parfois nettement interrompus à cause de l'impossibilité de raconter une actualité en devenir - c'est le cas d'Henriette et Richard (1792), des Lettres trouvées dans des portefeuilles d'émigrés (1793), de la suite de Trois Femmes -, à la fois matérielle et structurelle dans Sir Walter Finch et son fils William et sa suite (composés en 1799 et publiés posthumes, l'un en 1806 et l'autre dans les CEuvres complètes). D'autres textes à thèse tels Honorine d'Userche (1795) et Sainte Anne (1799) s'avèrent en revanche bel et bien terminés, traduisant par une forme plus traditionnelle l'intention de la romancière de développer ses thématiques en faisant coïncider « fin » et « finalité ».

4 C'est donc tout un art de la clôture et de son absence que déploie l'auteure. Son refus du «mot de la fin » irritait vivement Madame de Staël, qui lui exprimait sa désapprobation dans une lettre du 27 août 1793: "Je me suis intéressée vivement aux Lettres Neuchâteloises, mais je ne sais rien de plus pénible que votre manière de commencer sans finir. [...] Qu'est-ce qu'un roman appelé Mistress Henley, celui-là aussi est-il fait à moitié ? Vous abuseriez un peu du talent qu'il faut pour tourmenter ainsi » (EC, IV, 162) $)^{9}$. Deux siècles plus tard, cette «manière de commencer sans finir » devait être reconnue comme une belle marque d'originalité dans le panorama littéraire de l'époque par Lucia Omacini, qui évoque de la sorte cette revendication d'autonomie poétique et esthétique : «Il n'est probablement que Mme de Charrière qui puisse se réclamer de plein droit d'une poétique du non-fini: ses romans nous sont parvenus tantôt inachevés, tantôt hâtivement terminés $»^{10}$. Ce désir d'originalité s'avère finalement fort ressenti surtout dans le cas des premiers romans. Alors que la production plus tardive est axée sur une actualité sociopolitique qui catalyse l'attention de cette "aristocrate révolutionnaire ${ }^{11}$ à son tout début favorable à la Révolution, les textes composés entre 1784 et 1787 se veulent plus délicats et intimistes et présentent une telle unité de thèmes, de sujets, de situations et de formes que Sigyn Minier-Birk a été tentée de les lire comme les quatre volets d'un même ouvrage ${ }^{12}$. Ainsi que l'ont déjà souligné de nombreuses études féministes, auxquelles on doit en partie leur redécouverte critique des années 1980, ces «romans de l'échec $»^{13}$ explicitent les réflexions de l'écrivain sur la condition féminine de l'époque. Terminés au point de vue formel et diégétique, ils font preuve d'originalité par leur fin « ouverte » qui 
laisse les interrogations évoquées tout au long du récit sans réponse, escamotant par-là les clausules canoniques des romans sentimentaux. Les Lettres Neuchâteloises s'(in)achèvent sur le départ imprévu d'Henry Meyer, qui abandonne son amante Marianne sans pouvoir l'assurer ni de son retour ni du développement de leur liaison; incapable de trouver son bonheur avec un mari « de roman » parfait mais trop froid et raisonnable, l'héroïne des Lettres de Mistriss Henley décrit sa vie conjugale à une correspondante sans pour autant réussir à bien comprendre les causes d'un malheur qui l'épuise et l'induit à une probable crise nerveuse; dans la première partie des Lettres écrites de Lausanne, Cécile et sa mère quittent la ville suisse du titre sans que le lecteur sache si l'amour de la jeune fille pour Milord Edouard pourra se concrétiser. Parmi les ouvrages composés avant la tourmente révolutionnaire, le seul Caliste, deuxième partie du roman lausannois, propose une clôture « forte » et apparemment conventionnelle quant à l'« histoire [...] romanesque [...] autant que triste » (EC, VIII, 189) de William et de son amante, morte de chagrin à cause de l'irrésolution de celui-ci. Le héros retrace pour la veuve lausannoise une portion bien achevée de sa vie, mais le texte se termine sans fournir aucune réponse quant à la situation incertaine des deux Lausannoises, auxquelles il est pourtant concrètement adressé ${ }^{14}$.

5 Si la clôture de ce dernier roman est relativement redevable à son statut de suite et de récit rétrospectif nécessitant $a b$ origine un achèvement, l'ouverture des autres ouvrages mentionnés relève en revanche d'un choix de l'auteur, qui les considérait comme bel et bien achevés. En témoignent les signes paratextuels et la correspondance. La première édition anonyme des Lettres Neuchâteloises et l'« Avertissement » de Caliste présentent deux notes faisant allusion à la possibilité d'une continuation. Leur indétermination signale cependant la reprise ironique d'un procédé au but performatif fort usé plus que l'attestation d'une volonté réelle de les rouvrir. Dans le premier cas, l'incertitude concernerait l'existence même du manuscrit en question : «L'Éditeur de ces Lettres ne sait ni si elles ont une suite, ni, au cas qu'elles en aient une, s'il pourra se la procurer » (Notes aux Lettres Neuchâteloises, CEC, VIII, 611). Dans le deuxième, l'éventuelle continuation dépendrait surtout de l'intérêt que les lecteurs pourraient manifester - «Supposé que cette seconde partie soit aussi bien accueillie du public que l'a été la première, nous tâcherons de nous procurer quelques-unes des lettres que les personnes que nous avons fait connoître ont dû s'écrire depuis » (EC, VIII, 201) -, ce qui pervertit les fondements mêmes du topos de la fiction de la non fiction. À cet égard, Yvette Went-Daoust rappelle bien l'importance et l'évidence de la part d'ironie déployée par l'auteure à l'égard d'un poncif romanesque désormais très usé :

On a plus d'une fois dénombré les effets que le fameux manuscrit authentique dont se prévalent volontiers les écrivains des XVII et XVIII ${ }^{e}$ sont censés produire sur le lecteur. En tête de toutes les listes figure forcément l'impression que celui-ci éprouverait de lire le compte rendu d'expériences vécues. Or l'incomplétude du récit peut évidemment renforcer cette impression. Mme de Charrière tient compte du procédé dans la mesure où elle le pervertit ; c'est-à-dire où elle en fait un usage ironique ${ }^{15}$.

6 La fonction ironique du paratexte en question est en effet attestée par le remplacement de la note concernant le premier texte par un poème adressé aux Neuchâtelois et faisant fonction de "réponse au public», de paratexte conclusif signalant la fermeture de l'ouvrage à l'occasion de la deuxième édition ( $E C$, VIII, 89). Par ailleurs, dans la correspondance l'éventualité d'une continuation n'est évoquée que pour être immédiatement désavouée : 
J'avois bien une continuation des lettres neuchâteloises dans la tête, \& elle auroit été moins neuchâteloise, mais après que j'ai été tout à fait reconnue j'ai perdu courage. L'air de grande vérité qui a fait vrayment un peu illusion ici ne pouroit plus produire son effet, \& c'est cet effet que je voulois produire; on ne verroit plus que moi au lieu d'un honnête \& aimable jeune commis, \&c \&c. ( $E C$, II, 454).

Les Lettres de Mistriss Henley devaient sembler à Charrière également bien achevées, puisqu'elles présentent une clausule forte - l'allusion à une mort métaphorique, sinon physique, de l'héroïne - et que dans ses lettres elle ne mentionne jamais la possibilité d'une reprise. Ce qui n'est pas le cas du texte lausannois, dont le deuxième volet paraît trois ans après le premier et dont il existe d'autres tentatives de suite rapidement avortées $^{16}$. Dans une lettre du 20 février 1785, Salgas souscrivait de la sorte à l'intention de la romancière de composer une suite qui développerait pourtant une autre histoire que celle de Cécile : «Vous avez une fort bonne idée de vouloir nous faire l'histoire du mentor du jeune Lord. C'est un personnage intéressant, et qui va fort bien à côté de la mère de Cécile » (CEC, II, 456).

Nullement redevables d'une adhésion aux pratiques éditoriales de l'époque - il n'est d'ailleurs pas inutile de préciser que le seul Caliste avait joui d'une bonne fortune auprès du public -, ces fins " ouvertes » traduisent donc un choix poétique et esthétique précis directement lié à la contiguïté des thèmes développés. Pour Moser-Verrey, ces romans épistolaires «constituent une vigoureuse prise de parole car les héroïnes mises en scène questionnent tant les usages relevant de la tradition que les idéaux prônés par la philosophie. Il s'agit en fait de repenser le statut de la jeune femme sous l'Ancien Régime $»^{17}$. Cette explicitation est axée sur la représentation de l'impasse d'existences élevées par leur médiocrité et leur universalité à un statut paradigmatique : le sort de la neuchâteloise Marianne paraît aussi sombre que celui de la lausannoise Cécile ou des Anglaises Caliste et Mrs Henley ${ }^{18}$. En particulier, celle-ci est l'héroïne d'un ouvrage à la fin incontournable en ce qu'elle est déjà inscrite dans son propre paratexte : le titre indique suffisamment que Mistriss Henley ne pourra jamais être rien d'autre que la femme de son mari, alors que l'épigraphe tirée des Fables de La Fontaine - «J'ai vu beaucoup d'hymens, \&c. » ( $C E C$, VIII, p. 99) - et l'incipit « Je crois que beaucoup de femmes sont dans le même cas que moi » ( $E C$, VIII, p. 102) ne sont que trop explicites quant aux possibilités accordées à un bonheur conjugal considéré à l'époque comme le seul achèvement féminin possible. La position de «mineure" propre aux romancières contemporaines induit toutefois Charrière à composer en " tacticienne ", pour reprendre une formule célèbre de Letzer ${ }^{19}$, et à charger son écriture "imparfaite " d'un sens critique dans la mesure où celle-ci met implicitement en discussion un modèle littéraire traditionnel et l'idéologie qui le sous-tend. Ce modèle n'est pas tant celui de l'œuvre classique et de son achèvement, dont l'érosion silencieuse traverse tout le siècle, mais celui d'un roman sentimental au schéma fort codifié, ainsi que le rappelle N. Miller quand elle écrit que " the heroine's text is plotted within this ideologically delimited space of an either/or closure, within the conventional rhetoric of the sociolect ${ }^{20}$. L'adhésion au schéma canonique se révèlerait en effet peu efficace du point de vue thématique. Le mariage entre les amants des Lettres Neuchâteloises et des Lettres écrites de Lausanne s'afficherait comme un dénouement esthétiquement "plat» ainsi que peu susceptible de faire ressortir la problématique de la condition féminine, alors qu'une conclusion malheureuse et «triste » classerait les romans dans une tradition trop usée. De même, on ne saurait imaginer une fin appropriée et inédite pour les Lettres de Mistriss Henley. Une révolte ouverte de cette femme peu «raisonnable» - "Mais je ne suis pas une femme 
raisonnable, vous [son mari] \& moi l'avons vu trop tard » ( $E C$, VIII, 117) - n'est même pas envisageable; en revanche, une mort fort conventionnelle ne pourrait suggérer ni l'état d'épuisement physique et émotif qui est le sien - « Mon âme ni mon corps ne sont dans un état naturel. Je ne suis qu'une femme, je ne m'ôterai pas la vie, je n'en aurai pas le courage ; si je deviens mère, je souhaite de n'en avoir jamais la volonté ; mais le chagrin tue aussi. Dans un an, dans deux ans, vous apprendrez, je l'espère, que je suis raisonnable \& heureuse, ou que je ne suis plus » (EC, VIII, 122) - ni la dénonciation de l'institution du «mariage considéré [comme] une sorte de mort, le destin de l'enterrée vive ${ }^{21}$.

L'(in)achèvement représenterait donc pour l'auteure un moyen de protestation original dans la mesure où il se propose de miner les formules sur lesquelles repose le fonctionnement d'une société patriarcale, d'ouvrir une faille dans les canons classiques de composition et d'imposer un point de vue « autre » à toute une tradition: «je suis bien très aise de n'être sous aucune bannière et de courir librement le pays de la littérature. J'aime mieux dans ce genre les bandits que les enrégimentés ", écrivait la romancière les $1^{\mathrm{er}}-2$ novembre 1800 à Chambrier d'oleyres (EC, VI, 172). Laurent Versini a déjà fait remarquer que «tous les romans d'Isabelle de Charrière proposent des leçons, mais des leçons dictées par l'expérience, expérience de marginaux, d'émigrés, d'étrangers, qui vient ébranler les certitudes et le confort des gens installés ${ }^{22}$. Pour sa part, Annie Rivara évoquait l'extrême richesse d'une œuvre inachevée, qui témoigne de ce qu'elle est sans exclure ce qu'elle pourrait être ${ }^{23}$ : en refusant de conclure, en méconnaissant les solutions traditionnelles, l'écrivain affirme le droit de ses héroïnes à une identité qui ne saurait être limitée à celle de femme et de mère, comme le suggèrent les fins classiques des ouvrages d'une époque à laquelle «nulle [femme] n'échappe à l'impératif du mariage $»^{24}$. À la marginalité de l'expérience présentée correspond une création dans les marges, à l'« imperfection » de l'existence - d'après l'acception latine de perfectus comme achevé, accompli - une « imperfection » de sa représentation. À la " perfection » de la littérature majeure des "gens installés ", cette femme auteur - qui se trouve par là même dans une position de minorité civile -, partout étrangère et elle-même inachevée ${ }^{25}$ oppose l' "imperfection" d'une écriture se proposant de donner une voix au silence des minorités en respectant dans ses formes mêmes le caractère inaccompli de leur identité civile.

10 L'absence de la fin serait donc dans ces cas à lire comme le signe d'une écriture au féminin qui assume également une signification plus large et une dimension métalittéraire, la narration se présentant comme la mise en abîme d'une œuvre dont le sujet - les liaisons amoureuses insatisfaisantes des personnages - devient speculum. Dans les relations paradigmatiques exposées la femme est obligée au silence, à la passivité, à l'anonymat, tout comme le lecteur du roman sentimental traditionnel, relégué dans une position d'infériorité et d'inertie face à un texte parfait au sens esthétique et formel qui lui est imposé. Chez Charrière, les héroïnes sont invitées à faire preuve de la même participation active demandée à un lecteur obligé de creuser plus en profondeur pour saisir l'indicible d'une condition inégale dans les plis de l'écriture ou dans les silences, les absences et les ratures de l'ouvrage. Pour la femme comme pour son lecteur, l'auteur appelle donc à l'âge d'une pleine maturité, ainsi que le suggèrent Dennis Wood quand il fait allusion à sa volonté d'« éveiller notre sens critique » et à son désir d'« ouvrir une discussion plutôt que de la clore ${ }^{26}$ ou Yvette Went-Daoust, d'après laquelle la romancière compterait bien sur notre exercice critique : « Il n'est même pas improbable qu'elle ait compté sur une participation active de ses lecteurs. Le pacte romanesque 
ménage toujours chez elle un espace au non-dit. Mme de Charrière fait confiance à son lecteur pour combler les vides de la narration et ultimement pour imaginer un dénouement $»^{27}$.

11 Ces fins ouvertes et ambiguës - au sens étymologique du terme de " pousser d'un côté et de l'autre »- ne représentent en effet que la dernière «imperfection» du texte. Ce qui apparaît de façon particulièrement évidente dans les Lettres de Mistriss Henley, significativement considérées par le pasteur Chaillet comme un «aimable cruel petit livre, excellent en littérature, mais, selon moi, dangereux en morale à divers égards ${ }^{28}$. Leur (in)achèvement apparait comme le point ultime d'un refus de « dé-finition » qui se fait expression matérielle et presque graphique du silence et du vide d'une existence. La critique a déjà montré que l'adoption d'une parole apparemment minoritaire, innocente comme peut l'être celle du quotidien féminin - et superflue car accessoire et dénuée de toute importance événementielle est ici finalisée à mettre en discussion l'institution matrimoniale et à «subvertir la valeur de la raison abstraite pour comprendre ou organiser la société $»^{29}$. Partagée entre la doxa et son esprit peu raisonnable, entre la réalité subordonnée qui est la sienne et ses propres aspirations, l'héroïne est condamnée à une désagrégation progressive de son être et d'une parole ressentie comme de plus en plus inutile et inefficace. La volonté qui donne origine à la narration - le désir de rétablir la justesse des faits par le retracement de «l'histoire de [son] mariage, du temps qui l'a précédé » et la peinture de "[sa] vie telle qu'elle est aujourd'hui» ( $E C$, VIII, 102) n'engendre pas le récit juste et précis auquel on pourrait s'attendre. Après deux lettres fort détaillées et prétendument objectives, l'écriture se replie graduellement sur ellemême dans une spirale d'incomplétude et d'incompréhension. Ce qui préfigure déjà la capitulation d'un individu miné par le sentiment de sa culpabilité et de son inadéquation au rôle de femme d'un mari dont la "perfection " préétablie et l'achèvement excessif empêchent son bonheur : «ce séjour est comme son maître, tout y est trop bien ; il n'y a rien à changer, rien qui demande mon activité ni mes soins» (ECC, VIII, 118) $)^{30}$. La troisième lettre ne présente déjà plus la promesse de reprendre bientôt la narration. La quatrième, qui rend aussi compte du texte de soumission adressé à M. Henley, montre l'aphasie progressive conséquente à l'anéantissement de l'être par l'allusion à un brouillon envoyé « avec toutes ses ratures » et contenant " presqu'autant de mots effacés que de mots laissés » ( $E C$, VIII, 117). Cette reddition résignée, auquel il serait difficile d'ajouter quoi que ce soit, représente en effet le véritable "événement-clôture " de l'ouvrage ${ }^{31}$ et préfigure le silence final. Si la cinquième lettre se réduit à un communiqué de service de six lignes annonçant la grossesse d'une épistolière épuisée - «Vous n'en aurez pas davantage pour aujourd'hui » ( $E C$, VIII, 119) -, la dernière épître est traversée par un abandon réitéré de la plume et, par métonymie, de la communication, qui annonce le prochain (in)achèvement définitif de l'ouvrage : «Demain, si je le puis, je reprendrai la plume [...] mais je me suis si fort agitée en me les retraçant, que je suis obligée de poser la plume » (EC, VIII, 120-121).

12 "Chez Isabelle de Charrière, l'inachèvement est, sinon un art, du moins un processus cohérent, partie intégrante d'une poétique», résume fort justement L. Vanoflen ${ }^{32}$. Elément constitutif de sa poétique et de son écriture, cette pratique ne se résout finalement pas au seul refus de la fin dans sa double acception de manque de dénouement et de finalité, mais relève d'une recherche stylistique inscrite dans la narration elle-même . Caractérisée par une condition consubstantielle d'entre-deux, cette femme des Lumières était aussi sceptique qu'opposée à croire dans des «systèmes» qui à ses yeux ne 
pouvaient qu'« égarer méthodiquement » ( $E C, \mathrm{I}, 41)$. Dans la tentative d'échapper aux codes littéraires de son époque, elle nous offre donc une écriture et une narration où la parole est (re)valorisée par son absence même, où l'escamotage d'un lieu textuel important et encore ressenti comme obligatoire au XVIII ${ }^{\mathrm{e}}$ siècle - l'incomplétude romanesque des textes in-finis de la période peut bien détourner ad libitum l'idée même d'achèvement, mais elle n'en désavoue pas la nécessité théorique - assume une valeur critique. Forme et thèmes se répondent d'autant plus étroitement que le questionnement évoqué de la doxa littéraire acquiert de plus une valeur historique en ce qu'il marque un moment de passage entre deux courants littéraires - le classicisme et le romantisme - à l'égard desquels Charrière maintient, une fois de plus, une position d'entre-deux: son refus de la complétude classique s'accompagne également de la conscience de l'importance du modèle de la bonne volonté mimétique, de la nécessité de règles de composition bien établies et d'un travail incessant et acharné sur le texte.

«À tort ou à raison, je suis d'avis qu'il est permis de dire tout ce qui est vrai : \& de laisser aux auditeurs et aux lecteurs à tirer de toute vérité qui leur est présentée l'usage qui leur semblera raisonnable et utile, ce n'est pas à moi à trier pour les autres les vérités qui leur conviennent, c'est à eux à se les adapter » (EC, IX, 791-792), écrira dix années plus tard la Dame du Pontet dans un Texte sur Jan Praal, un brouillon composé à partir du personnage de l'instituteur matérialiste et athée des Trois Femmes. Ambiguë, neutre, imparfaite, (in)achevée : l'écriture de Charrière apparait finalement traversée par un appel constant à une réception critique active, ses textes demandant un rapport écrivain-lecteur dynamique car il est joué sur le déchiffrement des non-dits et des blancs du texte et sur le dépassement de son sens premier et plus traditionnel. Quelques années plus tard, dans la première partie de Sir Walter Finch et son fils William, cette femme des Lumières sceptique et exigeante devait réifier son désir d'une réception tout aussi sceptique et exigeante de la page écrite par l'évocation de Williams Bourg, une tour bâtie en Écosse en signe de continuité entre passé, présent et futur, au-dessous de laquelle sont ensevelis des livres et présentant une inscription à la signification fort explicite: «Dig. Deeper. Creusez. Profundius » (EC, IX, 559, italique dans le texte).

\section{NOTES}

1. D. Diderot, Supplément au Voyage de Bougainville, dans Id., Contes et Romans, M. Delon (éd.), avec la collaboration de J.-C. Abramovici et S. Pujol, Paris, Gallimard, "Bibliothèque de La Pléiade ", 2004, p. 579.

2. P. Zumthor, Babel ou l'inachèvement, Paris, Seuil, 1997, p. 221.

3. A. Rivara, « Synthèse ", dans A. Rivara et G. Lavorel (dir.), L'CEuvre inachevée. Actes du Colloque International (11 et 12 décembre 1998), Lyon, C.E.D.I.C, 1999, p. 272.

4. Cf. A.-K. Mortimer, La Clôture narrative, Paris, Corti, 1985, p. 77-108 et A. Viala, Naissance de l'écrivain. Sociologie de la littérature à l'âge classique, Paris, Minuit, 1985.

5. A.-F. Garreta, Fins de romans, XVII ${ }^{e}-X V I I I^{e}$ siècles. Stylistique, rhétorique, poétique d'un lieu textuel, thèse, New York, N.Y. University, 1988, p. 220. 
6. M. Moser-Verrey, Isabelle de Charrière : salonnière virtuelle. Un itinéraire d'écriture au XVIII ${ }^{e}$ siècle, Paris, Hermann, 2013.

7. G. Louÿs, Des romans inachevés. Étude historique et structurale, thèse, Paris, Université de Paris VII, 1998, p. 72.

8. I. de Charrière, Euvres Complètes, J.-D. Candaux, C. P. Courtney, P. H. Dubois, S. Dubois, P. Thompson, J. Vercruysse, D. M. Wood (éd.), Amsterdam-Genève, Van Oorschot, 1979-1984, t. II, p. 454 (dorénavant $\mathbb{C C}$, suivi du numéro du tome en chiffres romains et du numéro de la page en chiffres arabes, entre parenthèses dans le texte). Il faut signaler que l'attitude de dévalorisation quant à son propre talent ou à la qualité de sa production contenue dans la réplique de l'écrivaine n'est pas pour leurrer et s'insère dans une « rhétorique de la modestie, de la réticence ou de l'insignifiance " assez récurrente chez les femmes auteures de la période. Cf. C. BahierPorte et R. Jomand-Baudry, «Introduction », dans Id. (dir.), Écrire en mineur au XVIII siècle, Paris, Desjonquères, 2009, p. 16.

9. Dans une note à la lettre en question, l'éditeur de la correspondance de la fille de Necker précise aussi que «La critique s'adresse non seulement aux Lettres Neuchâteloises [...] mais aussi à Caliste, à la première partie des Lettres écrites de Lausanne, et à l'ouvrage dont il est question dans la phrase suivante. L'absence d'un dénouement bien défini contribue plutôt, selon nous, à la justesse et à la vérité de ces récits ", G. de Staël, Correspondance générale, B. W. Jasinski (éd.), Paris, Pauvert, 1962, t. II, p. 471.

10. L. Omacini, Le Roman épistolaire français au tournant des Lumières, Paris, Champion, 2003, p. 173.

11. I. Vissière et J.-L. Vissière (dir.), Isabelle de Charrière: une aristocrate révolutionnaire. Écrits (1788-1794), Paris, Des Femmes, 1988.

12. S. Minier-Birk, Madame de Charrière. Les Premiers romans, Paris-Genève, Slatkine, 1987, p. 88.

13. E. Leborgne, "Introduction ", dans I. de Charrière, Trois Romans d'Isabelle de Charrière : Lettres Neuchâteloises, Lettres de Lausanne, Honorine d'Userche, E. Leborgne (éd.), Saint-Étienne, Publications de l'Université de Saint-Étienne, 2011, p. 11.

14. Beaucoup de critiques découvrent toutefois une forte unité thématique entre ces deux sections. Pour J. Starobinski, l'histoire de Caliste répond bien au roman précédent, dont elle serait la "version dramatisée ", J. Starobinski, «Les Lettres écrites de Lausanne de Madame de Charrière: inhibition psychique et interdit social», dans M. Werner Krauss, R. Pomeau, R. Garaudy (dir.), Roman et lumières au XVIII ${ }^{e}$ siècle. Colloque décembre 1968, Paris, Éditions sociales, 1970, p. 133. S. Minier-Birk aussi (op. cit., p. 52) considère que les deux histoires sont parallèles dans la mesure où la tragédie de la jeune anglaise peut répondre aux questions de la mère de Cécile. La confession de William montrerait que beauté, bonté, charme ou talents ne sauraient pousser un homme faible et irrésolu à défier les préjugés sociaux et les conventions de sa propre classe.

15. Y. Went-Daoust, "Genres romanesques et modalités de l'inachèvement chez Madame de Charrière ", dans A. Rivara et G. Lavorel (dir.), op. cit., p. 200. La justesse de l'observation se révèle d'autant plus évidente si on compare ces paratextes à la note, bien plus conforme aux modèles des pierres d'attente de l'époque, insérée dans l'« Avis» de l'ouvrage de jeunesse Le Noble : «S'il nous parvient quelque autre production semblable de la même main, nous en ferons part au Public », ECC, VIII, 20.

16. Cf. CEC, VIII, 239-247.

17. M. Moser-Verrey, Isabelle de Charrière : salonnière virtuelle. Un itinéraire d'écriture au XVIII ${ }^{e}$ siècle, cit., p. 121. Voir aussi Id., «Isabelle de Charrière en quête d'une meilleure entente », dans Stanford French Review, 11, 1987, p. 76.

18. Pour S. Minier-Birk, ces existences se ressemblent au point qu'on est tenté de les lire comme la continuation l'une de l'autre : le mariage raisonnable et malheureux de Mrs Henley pourrait bien être celui auquel seront destinées Marianne ou Cécile, dont la mère ressemble à une Mrs Henley plus mûre qui n'a trouvé son bonheur que dans la maternité. S. Minier-Birk, op. cit., p. 88. 
19. Le critique met sur le compte de l'utilisation de tactiques le fait de «assuming fictional identities, treating serious matters in a light tone, never expressing unilaterally on any subject, and undermining the claims of her various opponents by putting them against each other ». J. Letzer, Intellectual Tacking. Questions of Education in the Works of Isabelle de Charrière, AmsterdamAtlanta, Rodopi, 1998, p. 6.

20. Cité par J. J. Allison, Revealing Difference. The Fiction of Isabelle de Charrière, London, Associated University Presses, 1995, p. 20.

21. Romans de femmes du XVIII siècle, R. Trousson (éd.), Paris, Laffont, 1996, p. 297.

22. L. Versini, Le Roman épistolaire, Paris, P.U.F., 1979, p. 188-189.

23. A. Rivara et G. Lavorel, « Avant-Propos », dans Id. (dir.), op. cit., p. 13.

24. Y. Went-Daoust, "Madame de Charrière et l'impératif du mariage », dans O. B. Cragg et R. Davison (dir.), Sexualité, mariage et famille au XVIII ${ }^{e}$ siècle, Montréal, Presses de l'Université Laval, 1998, p. 181.

25. P. Thompson résume bien la condition d'entre-deux qui est celle de la romancière : «la situation d'Isabelle de Charrière est un peu celle d'une émigrée. Elle est noble, mais se dépouille des privilèges de sa noblesse, corps dont elle a toujours soupçonné qu'il n'avait plus rien qui le justifiât dans chacun de ses membres; elle reste, en dépit de son mariage, une étrangère en Suisse ; elle s'intéresse encore passionnément aux Pays-Bas qu'elle juge et qu'elle a quittés. Elle est, avant la lettre, un spécimen artificiel de ce qu'aurait dû être l'émigrée future. La manière dont elle réagit à cette situation est d'installer dans ce malaise, dans cet entre-deux, comme eût dit Sainte-Beuve, l'originalité de sa réflexion sur les hommes et particulièrement sur ses contemporains ", P. Thompson, "Madame de Charrière et l'émigration », dans Lettre de Zuylen et du Pontet, 6, septembre 1981, p. 8.

26. D. Wood, «Isabelle de Charrière ou la difficulté de conclure », dans Ph. Baron, D. Wood, W. Perkins (dir.), Femmes et littérature. Colloque des Universités de Birmingham et Besançon, Besançon, Presses Universitaires Franc-Comtoises, 2003, p. 223.

27. Y. Went-Daoust, «Lettres écrites de Lausanne. «J'ai un foible pour mon sexe », dans Id. (dir.), Isabelle de Charrière. (Belle de Zuylen). De la correspondance au roman épistolaire, Amsterdam-Atlanta, Rodopi, 1995, p. 194.

28. Cité par Ph. Godet dans Id., Madame de Charrière et ses amis, Genève, Slatkine Reprints, 1973, t. I, p. 245.

29. Marta Figlerowicz, «Rester femme, devenir personne : l'externalisation dans les Lettres de Mistriss Henley ", dans Dix-Huitième Siècle, 42, 2010, p. 681. D'après le critique, l'externalisation des émotions et des pensées de l'héroïne sur les espaces domestiques est assimilable à la représentation des éléments du décor théâtral et permettrait de renverser l'idée même de l'existence d'une raison universelle, de rendre plus fiable une narratrice chaotique, de suggérer la dénonciation de l'institution matrimoniale. Cf. ibid., p. 680-683. Pour sa part, L. Mall retrouve les causes du malheur de la jeune anglaise dans l'antinomie opposant les riens de la domesticité et le « Tout » établi par les valeurs bourgeoises de raison et de nature : « Le texte est tout entier bâti sur une tension entre détail et totalité. [...] le monde domestique [...] est bien un domaine clos unifié par un dominus propre à domestiquer le contenu du domus. La fonte, l'absorption des êtres et des choses jusque dans le moindre détail dans cet ensemble est la condition et la conséquence nécessaires de son bon fonctionnement. Les détails proprement traités devraient y prendre leur place assignée - secondaire -, invisibles comme une femme de devoir. Mais ils ne sont dans le texte qu'aspérités, marques de l'impatience et du tort («ce sont de petites choses qui ... m'impatientent, et me font avoir tort»), du refus de souffrir et de marcher droit», L. Mall, «Perdue dans les détails: les Lettres de Mistriss Henley de Charrière et l'écriture de la désintégration ", dans Orbis Terrarum, 52, 1997, p. 180. Cf. aussi Y. Citton, «Trois fois rien : roman, galanterie, scrupule (Bougeant, Bastide, Charrière) ", dans J. Herman, A. Pachoud, P. Pelckmans 
et F. Rosset (dir.), L'Assiette des fictions. Enquêtes sur l'autoréflexivité romanesque, Louvain-la-Neuve, Peeters, 2010, p. 195-211.

30. Au tout début du texte Mistriss Henley explique qu'elle a pris la plume après la lecture du Mari Sentimental de Samuel de Constant, à propos duquel elle formule un jugement tristement prophétique : « cet homme-là [M. Bompré, le héros malheureux] eût grand tort, après tout, de se marier. Son bonheur, tout son sort, étoit trop établi ; sa femme n'avoit rien à faire qu'à partager des sensations qui lui étoient nouvelles \& étrangères; elle n'avoit point de Nanon, point d'Antoine, point d'Hector [les domestiques et le chien de son mari], point de voisins à rendre heureux, point de liaisons, point d'habitudes ; il n'y avoit pas là de quoi occuper une existence ", CEC, VIII, 101.

31. À propos du dénouement contradictoire des Mémoires de Prévost, Francis rappelle qu'il faut départager la conclusion de l'événement-clôture qui donne la véritable finalité du texte. Cf. R. A. Francis, «Les Fins de roman chez l'abbé Prévost », dans Id. et J. Mainil (dir.), L'Abbé Prévost au tournant du siècle, Oxford, The Voltaire Foundation, « SVEC $2000: 11$ », 2000, p. 135-136.

32. L. Vanoflen, "Mme de Charrière et l'inachèvement romanesque ", dans A. Rivara et G. Lavorel (dir.), op. cit., p. 197.

\section{RÉSUMÉS}

Malgré une adhésion théorique aux préceptes de l'esthétique classique, l'incomplétude sous-tend de façon importante la littérature romanesque du XVIII ${ }^{\mathrm{e}}$ siècle. La production d'Isabelle de Charrière s'avère en cela originale car l'absence de la fin assume une valeur critique pleinement revendiquée. Caractérisés par une contiguïté remarquable de thèmes, de sujets et de formes, les premiers ouvrages de l'auteure présentent une réflexion sur la position féminine de l'époque que la pratique de l'(in)achèvement et une conclusion «ouverte " contribuent à véhiculer dans la mesure où elles questionnent le schéma du roman sentimental ainsi que l'idéologie qui le soustend. En faisant appel à la participation critique du lecteur, l'imperfection (dans son acception latine de perfectus, accompli, achevé) de la narration et de l'écriture de Charrière acquiert une valeur critique et métalittéraire.

\section{INDEX}

Mots-clés : Charrière (Isabelle de), roman du XVIIIe siècle, inachèvement, Lettres Neuchâteloises, Lettres écrites de Lausanne, Lettres de Mistriss Henley 